\title{
A No-Depolarization Theorem for Rotator-Aided Resonance Crossing
}

\author{
Dennis W. Sivers \\ Portland Physics Institute, Portland, Oregon 97201 \\ and \\ Spin Physics Center, University of Michigan, Ann Arbor, MI 48109-1120
}

\begin{abstract}
An rf frequency rotator magnet provides a useful tool for manipulating particle spins in any accelerator or storage ring with polarized beams. This note briefly demonstrates the general idea of the rotator-aided crossing of spin resonance and sketches the proof of a general theorem about the process. The important question of whether this technique can be useful for a specific spin resonance at a particular accelerator involves a detailed analysis of the impact of the rotator on a range of machine parameters.
\end{abstract}

The use of coherent rotator magnets for the manipulation of particle spins in accelerators and storage rings has a venerable history. For example, in a series of experiments at IUCF [1], a group led by A. Krisch has conclusively demonstrated the value of both rf-dipole and rf-solenoid rotators. It is particularly important to note the results of the Brookhaven accelerator group reported at this meeting [2], involving the use of an rf-dipole magnet to aid in the crossing of intermediate-strength intrinsic resonances in the AGS.

Instead of dealing with a specific situation, it is constructive to first consider a general theorem involving the use of rotators. For this purpose, a rotator will be defined as a device which produces a localized magnetic field intersecting the beam as given by,

$$
\vec{B}_{i}=\hat{e}_{i}\left(C_{m} \cos v_{m} t\right)
$$

In this context it will be assumed that the magnet parameters can be adjusted independently and are controllable on a time scale that is long compared to $v_{m}^{-1}$. That is, we can write

$$
\begin{aligned}
& C_{m}=C_{m}(t) \\
& v_{m}=v_{m}(t)
\end{aligned}
$$

and vary these parameters to aid in resonance crossing. It is important for this discussion that it is possible to vary $C_{m}$ over a sufficient range to produce an induced spin resonance capable of generating a spin flip.

We can think of a spin resonance as an operator that impacts the polarization of a beam when the spin tune, $v_{s}(t)$, passes the resonance frequency. The strength of a resonance is important. If a spin resonance is sufficiently weak, it is possible to cross 
the resonance rapidly with little or no depolarization. Conversely, if it is sufficiently strong, it is possible to use the Froissart-Stora [3] technique of adiabatic crossing to induce a spin flip. Measuring the beam polarization before and after crossing, we label the two limits

$$
\begin{aligned}
& P_{f} / P_{i} \cong 1 \\
& P_{f} / P_{i} \cong-1
\end{aligned}
$$

as "controlled" crossings since they do not destroy beam polarization. Those resonances which are dangerous to polarization are of intermediate strength and do not allow "controlled" crossings.

A rotator magnet operating as described above creates an induced spin resonance at $v_{m}(t)$ with a strength that can be varied from weak to strong depending on the value of $C_{m}(t)$. With these preliminaries, we can formulate a no-depolarization condition with the following theorem.

Theorem. Assume that a spin resonance, $v_{\text {res }}$, divides the plane $\left\{v \in\left[v_{\min }, v_{\max }\right]\right.$ and $\left.t \in\left[t_{\min }, t_{\max }\right]\right\}$ into two disjoint regions so that it is not possible to form a path, $v_{s}(t)$, that passes from $v_{s}\left(t_{\text {min }}\right) \leq v_{\text {res }}$ to $v_{s}\left(t_{\max }\right) \geq v_{\text {res }}$ without crossing the resonance. By adding an additional, induced, resonance at $v_{m}(t)$ to the system, the $\{v, t\}$ plane is separated by the two resonances into three disjoint regions. Varying the magnet parameters $C_{m}(t)$ and $v_{m}(t)$ for the induced resonance allows a path $v_{s}(t)$ that navigates from $v_{s}\left(t_{\min }\right) \leq v_{\text {res }}$ to $v_{s}\left(t_{\max }\right) \geq v_{\text {res }}$ with either no resonance crossings or two "controlled" resonance crossings.

The proof of this simple theorem utilizes quantum- mechanical operator mixing and the fact that the interference of the operators prevents an overlap of two resonances so that the $\{v, t\}$ plane is cut by the two resonance lines as indicated in Fig. 1. In addition, in regions where operator mixing is important, the interference effects that mix the operators assure that both resonances will have similar strength. Varying the magnet parameters therefore allows control location of the induced resonance and of the strength of both resonances over a finite region of the $\{v, t\}$ plane. Coordination of the acceleration path $v_{s}(t)$ with the variation of the magnet parameters therefore allows a path which either avoids the "mixed" resonances or executes two "controlled" crossings.

To convert this from a simple mathematical plane-cutting theorem to a physics "no depolarization" theorem requires a considerable understanding of the impact of the operation of the rotator magnet on other accelerator parameters. The theorem cannot guarantee that the technique will be practical. Unlike the operation of a Siberian Snake [4], the use of a coherent rotator magnet merely aids in the crossing of a resonance; it does not eliminate a class of resonances. Application of the technique requires testing and polarization measurements to achieve optimal results. Because of the time required to perform a polarization measurement, this can be a serious obstacle. However, once a successful resonance-crossing strategy has been determined, it can be successfully repeated without further input from polarization measurements. The experiments at IUCF have shown that adiabatic spin flips through controlled resonance crossings require tuning efforts. Avoiding a resonance crossing does not guarantee that there will be no depolarization. Because of spin-tune "spread" and other effects, 
operating a beam near a resonance for a finite amount of time can lead to depolarization just like a resonance crossing. The results of the Brookhaven group justify the use of resonance crossings.[2] The techniques of varying the magnet parameters outlined here merit further study but do not guarantee results.

\section{ACKNOWLEDGEMENTS}

The co-chairs of this conference, A. Krisch and T. Roser, created a collaborative atmosphere that was very encouraging to new ideas, and the hospitality of the Spin Physics Center at Michigan was very nourishing. As a result, the author received considerable aid from suggestions by Ya. Derbenev, L. Teng and M. Bai.
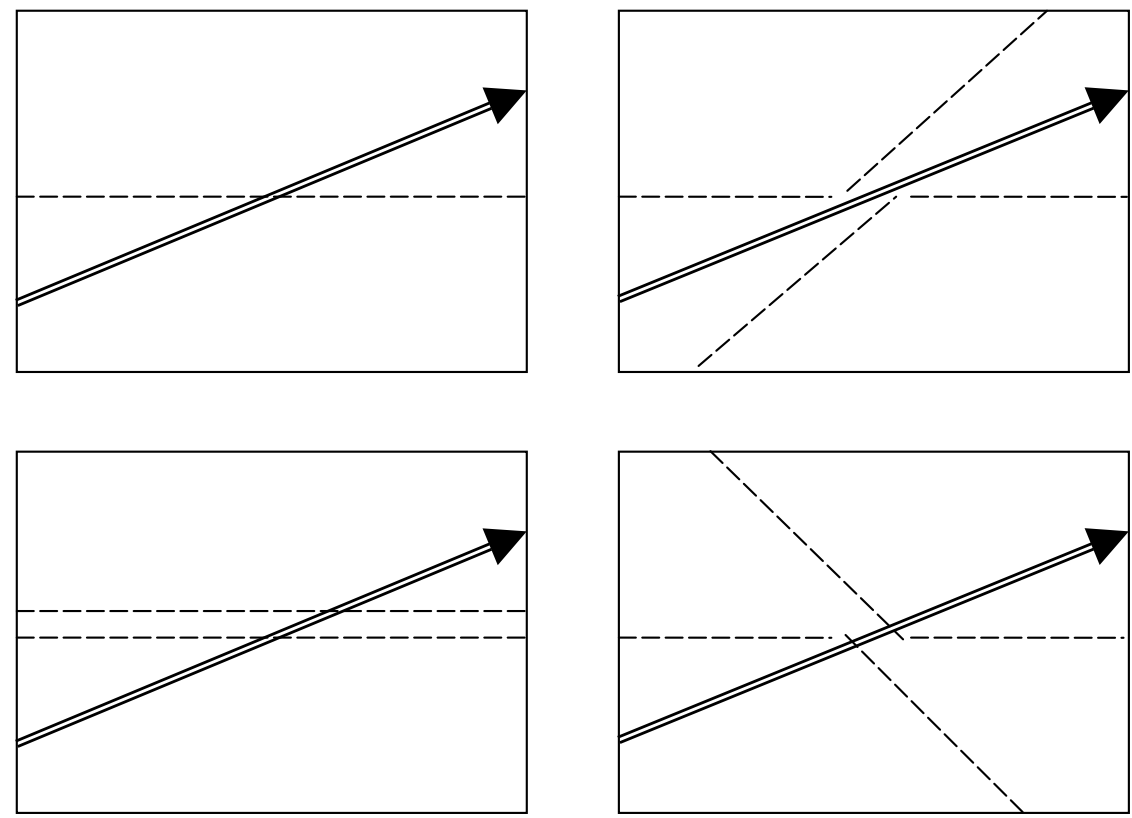

FIGURE 1. Four sketches of the tune vs. time plane with different resonance configurations. Top left, one resonance. Top right, induced resonance with increasing frequency. Bottom left, induced resonance with fixed frequency close to intrinsic resonance. Bottom right, induced resonance with decreasing frequency. Arrows indicate possible path for spin tune of ring.

\section{REFERENCES}

[1.] See, for example, B. Blinov et al., Phys. Rev. Lett. 81, 2906 (1998); V. Anferov et al., Phys Rev ST-AB 3, 041001 (2000); B. Blinov et al., Phys Rev ST-AB 3, 104001 (2000).

[2.] M. Bai, these proceedings. See, also, M. Bai and T. Roser, "Crossing a coupling spin resonance with an RF dipole" in Proceedings of the $14^{\text {th }}$ Int'l Spin Physics Symposium, Osaka, Japan, October 2000, edited by K. Hatanaka et al., AIP Conference Proceedings 570, New York (2001), p. 741.

[3.] M. Froissart and R. Stora, Nucl. Instrum. Methods 7, 297 (1960).

[4.]Ya. Derbenev and A. Kondratenko, Sov. Phys. JETP 37, 968 (1973). 